\title{
Caracterización botánica y anatómica de 5 especies maderables de bosque secundario en el sector el Rocío, del Cantón Loreto, provincia de Orellana
}

\author{
Botanical and anatomical characterization of 5 timber species of \\ secondary forest in the sector El Rocío, from Loreto canton, province of \\ Orellana
}

Eduardo Patricio Salazar Castañeda. ${ }^{1}$, Carlos Francisco Carpio Coba. ${ }^{2}$ Vilma Fernanda Noboa Silva. ${ }^{3}$ \& Izamar Vanessa Verduga Coyago. ${ }^{4}$

Recibido: 11-01-2021 / Revisado: 16-01-2021 /Aceptado: 10-02-2021/ Publicado: 05-03-2021

\begin{abstract}
.
DOI: https://doi.org/10.33262/concienciadigital.v4i1.2.1584

Introduction. This research helps to solve some of the urgent needs of the Ecuadorian forestry sector, in order to implement a set of strategies that serve for the identification, botanical and anatomical characterization of timber species, to help reduce the problems of illegal logging and logging. improve mobilization and marketing processes in the territory. At the same time, it serves as the basis for further research related to mechanical properties, physical properties, durability and protection of economically important species. Objective. To determine macroscopic and microscopic characteristics of Apeiba membranacea, Cordia alliodora, Erisma uncinatum, Jacaranda copaia and Virola glycycarpa. Methodology. For the development of the research, the samples were

\footnotetext{
${ }^{1}$ Escuela Superior Politécnica de Chimborazo, Facultad de Recursos Naturales. Carrera de Ingeniería Forestal. Riobamba, Ecuador, eduardo.salazar@espoch.edu.ec. Código ORCID: 0000-0001-7737-5415.

2 Escuela Superior Politécnica de Chimborazo, Facultad de Recursos Naturales. Carrera de Ingeniería Forestal. Riobamba, Ecuador, ccarpio@espoch.edu.ec. Código ORCID: 0000-0002-7361-7664.

${ }^{3}$ Escuela Superior Politécnica de Chimborazo, Facultad de Recursos Naturales. Carrera de Ingeniería Forestal. Riobamba, Ecuador. vilma.noboa@ espoch.edu.ec. Código ORCID: 0000-0002-3164-7304.

${ }^{4}$ Investigadora Forestal (GIFOR). Riobamba, Ecuador. izamita.94@ gmail.com. Código ORCID: 00000002-4282-1822.
} 
identified in the herbarium of the Escuela Superior Politécnica de Chimborazo. To describe the organoleptic properties, a sample with a size of $15 \times 8 \times 2 \mathrm{~cm}$ was obtained and its brightness, color, grain, smell, taste, texture, and marbling were analyzed. For the study of the anatomical characteristics, $2 \times 2 \times 2 \mathrm{~cm}$ cubes were prepared to soften them, each sample was placed in a glass bottle with distilled water. The flask was covered with aluminum foil and autoclaved at a temperature of $121^{\circ} \mathrm{C}$ and a pressure of 1.1 atmospheres. Three planes were made with a rotary microtome: transverse, radial and tangential. To observe the anatomical structures of the sections, safranin, Astrablue and Astrablue / Safranin were used. To calculate the density, each sample was weighed and a calibrator was used to calculate the volume. To obtain the $\mathrm{pH}$ value, 10 grams of sawdust (substrate) were weighed, which was placed in a beaker with distilled water to form a homogeneous mixture and then the $\mathrm{pH}$ value was measured. Results. Astrablue / Safranin staining is the one with the best performance. Each species presented macroscopic characteristics different from each other. According to the smell and taste, three of them have the same characteristics, while for the grain the five species have the same type. Regarding the brightness, they all have it of medium type. Regarding the size of the pores, almost all the species presented medium pores, except Jacaranda copaia, which presented large pores. In relation to the number of pores in Jacaranda copaia the least number of pores was observed, while in Erisma uncinatum the highest amount. Virola glycycarpa has the highest density and Membranous Apeiba the lowest. Two of the studied species presented basic $\mathrm{pH}$ and three acidic $\mathrm{pH}$. Conclusions. The species belonging to five different families presented different characteristics.

Keywords: Timber species, macroscopic and microscopic characteristics, plant tissues, pores.

\section{Resumen.}

Introducción. Esta investigación ayuda a solventar algunas de las necesidades urgentes del sector forestal ecuatoriano, con el fin de implementar un conjunto de estrategias que sirva para la identificación, caracterización botánica y anatómica de las especies de maderables, para ayudar a reducir los problemas de tala ilegal y mejorar procesos de movilización y comercialización en el territorio. Al mismo tiempo, sirve como base para continuar con investigaciones más profundas relacionadas con las propiedades mecánicas, las propiedades físicas, la durabilidad y protección de especies de importancia económica. Objetivo. Determinar características macroscópicas y microscópicas de Apeiba membranacea, Cordia alliodora, Erisma uncinatum, Jacaranda copaia y Virola glycycarpa. Metodología. Para el desarrollo de la investigación, las muestras fueron identificadas en el herbario de la Escuela Superior Politécnica de Chimborazo. Para describir las propiedades organolépticas, se obtuvo una muestra con un tamaño de 15 x 8 x $2 \mathrm{~cm}$ y se analizó su brillo, color, grano, olor, sabor, textura, y veteado. Para el estudio de las características anatómicas se prepararon cubos de 2×2×2 cm para ablandarlos, cada muestra se colocó en una botella de vidrio con agua destilada. El frasco se cubrió con 
papel de aluminio y se lo llevó al autoclave a una temperatura de $121{ }^{\circ} \mathrm{C}$ y a una presión de 1,1 atmósferas. Se realizaron tres planos con un micrótomo rotatorio: transversal, radial y tangencial. Para observar las estructuras anatómicas de los cortes se utilizaron la safranina, el Astrablue y Astrablue / Safranina. Para calcular la densidad se pesó cada muestra y se usó un calibrador para calcular el volumen. Para obtener el valor de $\mathrm{pH}$ se pesaron 10 gramos de aserrín (sustrato) que se colocó en un vaso de precipitación con agua destilada para formar una mezcla homogénea y luego se procedió a medir el valor de pH. Resultados. La tinción con Astrablue / Safranina es la que mejor desempeño mostró. Cada especie presentó características macroscópicas diferentes entre ellas. De acuerdo al olor y sabor, tres de ellas presentan características iguales, mientras que para el grano las cinco especies presentan el mismo tipo. En lo referente al brillo todas lo presentan de tipo mediano. En cuanto al tamaño de los poros casi todas las especies presentaron poros medianos, excepto Jacaranda copaia que presentó poros grandes. En relación a la cantidad de poros en Jacaranda copaia se observó la menor cantidad de poros, mientras que en Erisma uncinatum la mayor cantidad. Virola glycycarpa presenta la densidad más alta y Apeiba membranácea la más baja. Dos de las especies estudiadas presentaron $\mathrm{pH}$ básico y tres $\mathrm{pH}$ ácido. Conclusiones. Las especies pertenecientes a cinco familias diferentes presentaron características diferentes.

Palabras claves: especies maderables, características macroscópicas y microscópicas, tejidos vegetales, poros.

\section{Introducción.}

Cuando se trabaja en el campo, la identificación, caracterización y reconocimiento de las especies maderables es muy importante, debido a que cada tipo de madera tiene una fenología, así como características físicas y químicas de la madera diferentes.

Aunque los seres humanos han utilizado la madera para muchos fines humanos, fundamentalmente la madera es una estructura biológica compleja compuesta por muchos compuestos químicos y tipos de células que interactuando de manera coordinada pueden ayudar a satisfacer las necesidades de las plantas. (Wiedenhoeft y Miller, 2005). Los troncos de los árboles realizan tres funciones importantes: soporte mecánico de las hojas que van a realizar la fotosíntesis (Rowe y Speck, 2005); reserva de agua, hidratos de carbono y otros nutrientes (Sauter y van Cleve, 1994); y el acarreo de agua y otras sustancias desde el suelo a las hojas (Sperry, 2003).

Algunas especies maderables han sido utilizadas desde hace tiempo atrás en la construcción, el aumento de su demanda hoy puede incrementar su valor comercial. En las regiones tropicales y subtropicales, hay áreas en donde existen especies con un elevado valor comercial y ecológico. No obstante, para aprovechar correctamente la madera, es necesario conocer sus características anatómicas, físicas y mecánicas (Agila, et al. 2018: p. 15).

Derivado de la falta de información sobre las características anatómicas, físicas, mecánicas y, químicas de la madera en Ecuador, no a sido posible implementar un 
apropiado procesamiento e industrialización de la madera lo que ha traído pérdidas económicas y ambientales al sector.

Es por ello que, para poder mantener nuestros bosques, es importante conocer las características de la madera de las especies que se encuentran en su interior, para poder hacer un mejor uso de ella, debido a que el mercado actual exige madera de excelente calidad. (Crespo, 2012: pp. 41-53).

\section{Metodología.}

\section{Identificación de las especies}

Las muestras utilizadas en esta investigación se obtuvieron del bosque secundario de la subregión El Rocío de la localidad de Loreto, Orellana. Se recolectaron muestras de las plantas y de la madera de de Apeiba membranacea, Cordia alliodora, Erisma uncinatum, Jacaranda copaia y Virola glycycarpa.

Las muestras de cada especie fueron herborizadas e identificadas en el Herbario de la Escuela Superior Politécnica de Chimborazo (CHEP).

Se prepararon cubos de madera siguiendo las normas (COPANT, 1972: 458-462).

\section{Características macroscópicas}

Las probetas de madera que se utilizaron para los estudios de las características macroscópicas tenían las siguientes dimensiones: 2 x 9 x $15 \mathrm{~cm}$ de espesor, ancho y largo respectivamente. Se utilizó la tabla de Munsell para determinar los colores de duramen y albura; el olfato para los olores; el gusto para los sabores y el tacto para identificar la textura.

\section{Análisis de características microscópicas en laboratorio}

\subsection{Ablandamiento de especies}

En esta etapa, se toma el cubo de 2 × 2 x $2 \mathrm{~cm}$ y se los ubica en un envase de vidrio y se lo cubre con agua destilada. El envase cubierto se lo coloca en el autoclave a una temperatura de $121^{\circ} \mathrm{C}$ y una presión de $1,1 \mathrm{~atm}$. Una vez finalizado el ciclo, se comprueba cuan blanda está la muestra. El número de ciclos que se empleen dependerá de la dureza de la madera. Para este estudio fue necesario un solo ciclo para todas las especies.

\subsection{Laminado de especies}

Una vez preparadas las muestras, se procedió a realizar los cortes transversal, radial y tangencial con la ayuda de un micrótomo como lo recomienda (Feijoo, 2019). El grosor de cada corte fue de $0,3 \mu \mathrm{m}$. Al final cada uno de los cortes fueron colocados en una placa de Petri.

\subsection{Tinturado de láminas}


Se coloca $20 \mathrm{ml}$ de safranina las láminas que están en la caja Petri y se deja actuar durante 1 minuto, Para la tinción con Astra blue se añadió $20 \mathrm{ml}$ y durante 3 minutos se deja actuar. Posteriormente con la ayuda de papel absorbente se retira el exceso de la solución.

Se preparó la solución de azafrán / Astra en una proporción de 1: 1, luego la solución se coloca sobre los cortes ubicados en una placa de Petri y se dejó reposar por 8 minutos, posteriormente se retiró el exceso de solución con la ayuda de agua destilada, finalmente se sumergió la placa en etanol al $96 \%$ durante tres minutos.

Con un microscopio utilizando los lentes ópticos 10x y 4x, se procedió a observar la anatomía de las muestras. Posteriormente se hicieron fotografías de las estructuras observadas de los tres tipos de corte.

\subsection{Análisis estadístico}

Para la evaluación del número de poros solitarios y poros múltiples de las cinco especies estudiadas se utilizó un Diseño completamente al azar (DBCA). Se usó un ANOVA para analizar los datos; Se aplicó la prueba de Shapiro-Wilks para comprobar la normalidad de los datos. En los casos en que hubo diferencias estadísticas entre los tratamientos se aplicó la prueba de Tukey ( $\mathrm{P}<0.05)$. Los análisis se hicieron con el programa estadístico Infostat (Di Rienzo et al. 2011).

\section{Resultados.}

1. Identificación realizada en el Herbario (CHEP).

Tabla 1 Identificación de las especies estudiadas

\begin{tabular}{lll} 
Familia & Nombre científico & Nombre Común \\
\hline Boraginaceae & Cordia alliodora (Ruiz \& Pav.) Cham. & Laurel \\
\hline Tiliaceae & Apeiba membranacea Spruce ex Benth & Peine de mono \\
\hline Myristicaceae & Virola glycycarpa Ducke & Sangre de gallina \\
\hline Bignoniaceae & Jacaranda copaia & Jacaranda \\
\hline Vochysiaceae & Erisma uncinatum Warm & Arenillo
\end{tabular}

Fuente: Equipo técnico (2020)

Las especies identificadas pertenecen a 5 familias diferentes, son comercializadas en el país y están sujetas a una gestión comercial y medioambiental activa a través del Ministerio del Medio Ambiente.

\section{Características macroscópicas}


La siguiente tabla resume los resultados de las características macroscópicas de la madera estudiada:

Tabla 2 Características macroscópicas de las especies estudiadas

\begin{tabular}{|c|c|c|c|c|c|}
\hline $\begin{array}{c}\text { Característica } \\
\text { s }\end{array}$ & $\begin{array}{l}\text { Cordia } \\
\text { alliodora }\end{array}$ & $\begin{array}{c}\text { Apeiba } \\
\text { membranace } \\
a\end{array}$ & $\begin{array}{c}\text { Virola } \\
\text { glycycarpa } \\
\text { Ducke }\end{array}$ & $\begin{array}{c}\text { Jacaranda } \\
\text { copaia }\end{array}$ & $\begin{array}{c}\text { Erisma } \\
\text { uncinatum }\end{array}$ \\
\hline Corteza & $\begin{array}{l}\text { Color pardo } \\
\text { grisáceo, } \\
\text { finamente } \\
\text { fisurada }\end{array}$ & $\begin{array}{c}\text { Color marrón } \\
\text { claro a } \\
\text { grisáceo, con } \\
\text { presencia de } \\
\text { lenticelas }\end{array}$ & $\begin{array}{l}\text { Color } \\
\text { marrón y } \\
\text { fisurada }\end{array}$ & $\begin{array}{l}\text { Color gris } \\
\text { claro, } \\
\text { ligerament } \\
\text { e fisurada }\end{array}$ & $\begin{array}{c}\text { Color gris } \\
\text { oscura, fina } \\
\text { y dura }\end{array}$ \\
\hline Color albura & $\begin{array}{l}\text { Marrón muy } \\
\text { pálido }\end{array}$ & $\begin{array}{l}\text { Marrón muy } \\
\text { pálido }\end{array}$ & $\begin{array}{c}\text { Marrón } \\
\text { fuerte }\end{array}$ & $\begin{array}{l}\text { Amarillo } \\
\text { pálido }\end{array}$ & $\begin{array}{c}\text { Marrón } \\
\text { amarillento } \\
\text { claro }\end{array}$ \\
\hline $\begin{array}{c}\text { Color } \\
\text { duramen }\end{array}$ & $\begin{array}{c}\text { Marrón } \\
\text { amarillento } \\
\text { oscuro. }\end{array}$ & $\begin{array}{c}\text { Marrón } \\
\text { amarillento }\end{array}$ & Marrón claro & Amarillo & Marrón rojizo \\
\hline Olor & $\begin{array}{l}\text { Agradable } \\
\text { no distintivo }\end{array}$ & $\begin{array}{l}\text { Agradable no } \\
\text { distintivo }\end{array}$ & Ausente & Ausente & Ausente \\
\hline Sabor & $\begin{array}{l}\text { Agradable } \\
\text { no distintivo }\end{array}$ & $\begin{array}{l}\text { Agradable no } \\
\text { distintivo }\end{array}$ & Ausente & Ausente & Ausente \\
\hline Grano & Recto & Recto & $\begin{array}{l}\text { Recto algo } \\
\text { entrecruzad } \\
\text { o }\end{array}$ & Recto & Recto \\
\hline Textura & Fina & Gruesa & Gruesa & Gruesa & Mediana \\
\hline Brillo & Medio & Medio a alto & $\begin{array}{l}\text { Medio a } \\
\text { opaco }\end{array}$ & Medio & Medio a bajo \\
\hline Veteado & $\begin{array}{c}\text { Arcos } \\
\text { superpuesto } \\
\text { s }\end{array}$ & $\begin{array}{c}\text { Arcos } \\
\text { superpuestos }\end{array}$ & $\begin{array}{l}\text { Jaspeado } \\
\text { satinado }\end{array}$ & Jaspeado & $\begin{array}{c}\text { Arcos } \\
\text { superpuesto } \\
\text { s }\end{array}$ \\
\hline
\end{tabular}

Fuente: Equipo técnico (2020)

\section{Características microscópicas de las cinco especies de importancia forestal}

La siguiente tabla resume los resultados del análisis microscópico de la madera de las especies estudiadas. 
ISSN: 2600-5859

Www.concienciadigital.org

Vol. 4, Nº1.2, p. 131-146, marzo, 2021

Tabla 3 Características microscópicas de las especies estudiadas

\begin{tabular}{ccccc}
\hline Especies & Poros & Parénquima & Radio & Porosidad \\
\hline Cordia alliodora & Solitarios & Paratraqueal & No & Difusa \\
Fig.1, Fig. 6, Fig. & radiales y & vasicéntrico y & estratificados & \\
11 & múltiples & paratraqueal & & \\
& radiales y & unilateral & &
\end{tabular}

racemiformes

de 2 y 3

\begin{tabular}{ccccc}
\hline Apeiba & Múltiples & Parénquima & Estratificados & Difusa \\
membranácea & radiales 2 y 3 & paratraqueal & & \\
Fig. 2, Fig. 7, Fig. & seguido de & vasicéntrico y & & \\
12 & solitarios & apotraqueal & & \\
\hline $\begin{array}{c}\text { Virola glycycarpa } \\
\text { Ducke }\end{array}$ & Múltiples & Paratraqueal & No & Difusa \\
\end{tabular}

Fig.3, Fig. 8, Fig. y 4, muy pocos

13

solitarios

Jacaranda

Solitarios,

Paratraqueal

No

Difusa

copaia

múltiples de $2 \mathrm{a}$

aliforme,

estratificados

Fig.4, Fig. 9, Fig.

3.

aliforme

14

confluente de

ala fina corta $y$

larga

$\begin{array}{ccccc}\begin{array}{c}\text { Erisma } \\ \text { uncinatum }\end{array} & \begin{array}{c}\text { Solitarios, } \\ \text { múltiples de 2 a }\end{array} & \begin{array}{c}\text { Parénquima en } \\ \text { bandas anchas }\end{array} & \begin{array}{c}\text { No } \\ \text { estratificados }\end{array} & \text { Difusa }\end{array}$

Fig.5, Fig. 10, Fig.

3

$y$ largas

15

Fuente: Equipo técnico (2020)
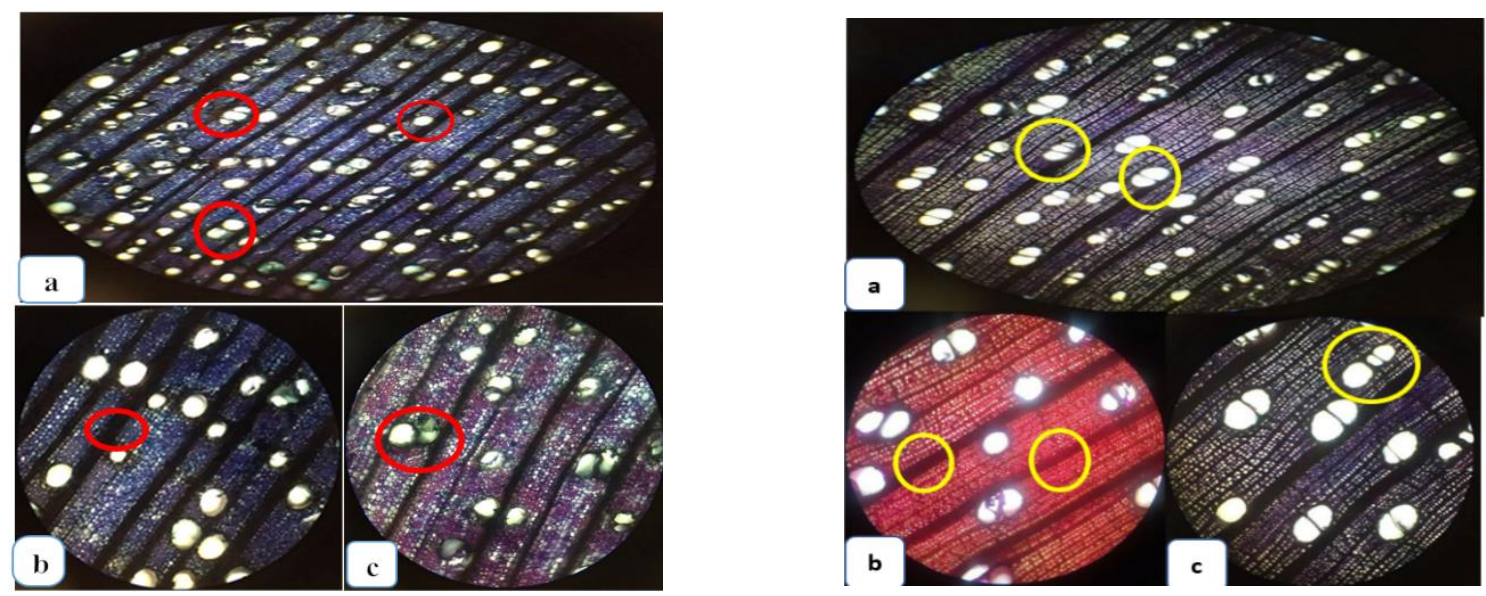
Figura 1. Vista en corte trasversal de la madera de Cordia alliodora a. poros solitarios radiales y múltiples radiales y racemiformes de 2 y 3 . b. radios finos y gruesos no estratificados. c. Poros con tílide

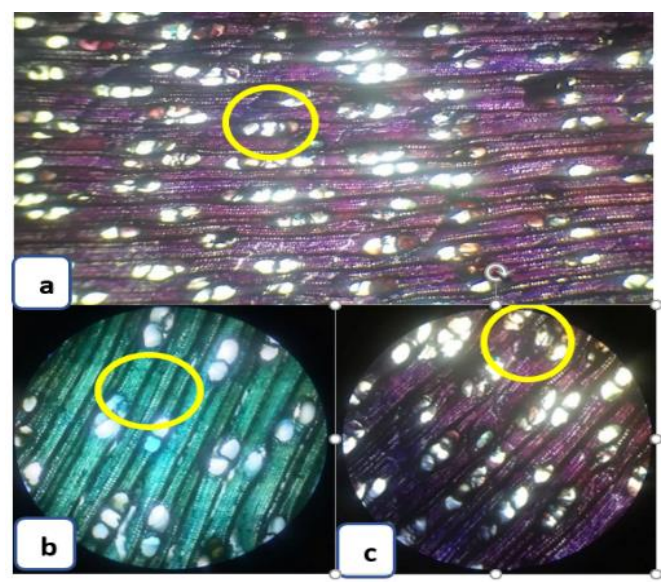

Figura 3. Vista en corte trasversal de la madera de Virola glycycarpa. a. poros múltiples radiales de 2,3 y 4 , muy pocos solitarios, b. radios finos. c. Poros ovalados algunos con gomas
Figura 2. Vista en corte trasversal de la madera de Apeiba membranacea a. poros solitarios y múltiples radiales de 2 y 3 . b. radios finos y gruesos estratificados. c. poros redondos y ovales

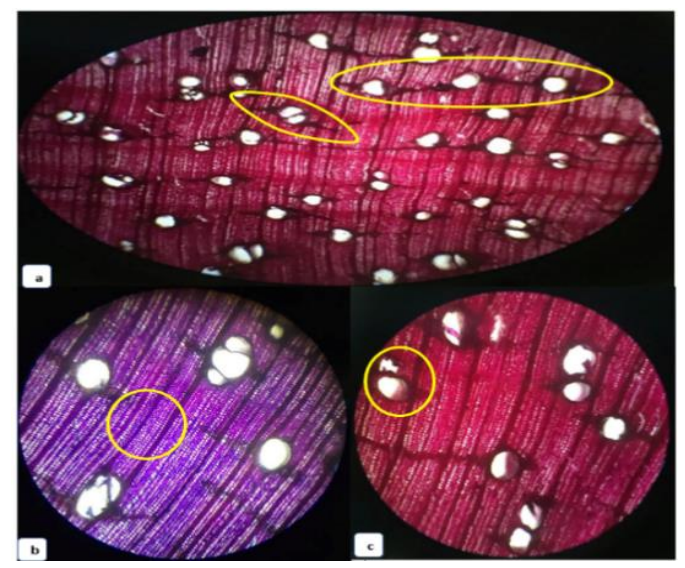

Figura 4. Vista en corte trasversal de la madera de Jacaranda copaia. a. poros solitarios, pocos múltiples de 2 a 3 , parénquima paratraqueal aliforme, aliforme confluente de ala fina corta y larga, porosidad difusa, b. radios muy finos $\mathbf{c}$. presencia de tílide.

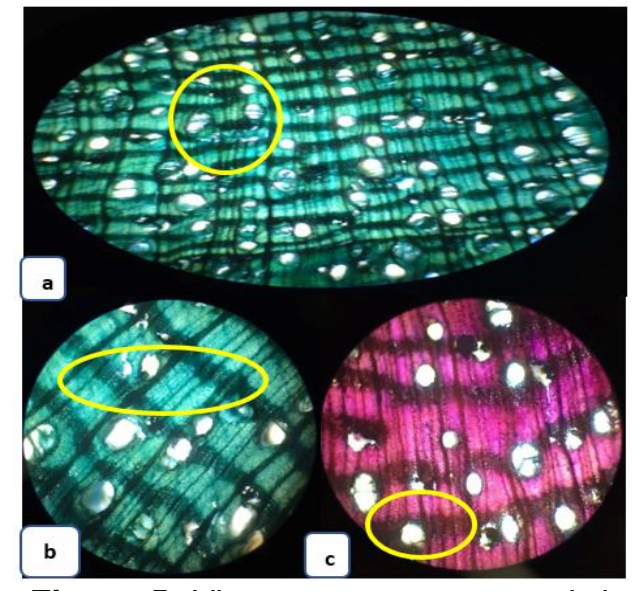

Figura 5. Vista en corte trasversal de madera de Erisma uncinatum. a. poros solitarios y múltiples de 2 a 3 , parénquima en bandas anchas y largas, porosidad difusa. b. radios 


\section{[DDigital}

ISSN: 2600-5859

wWw.concienciadigital.org

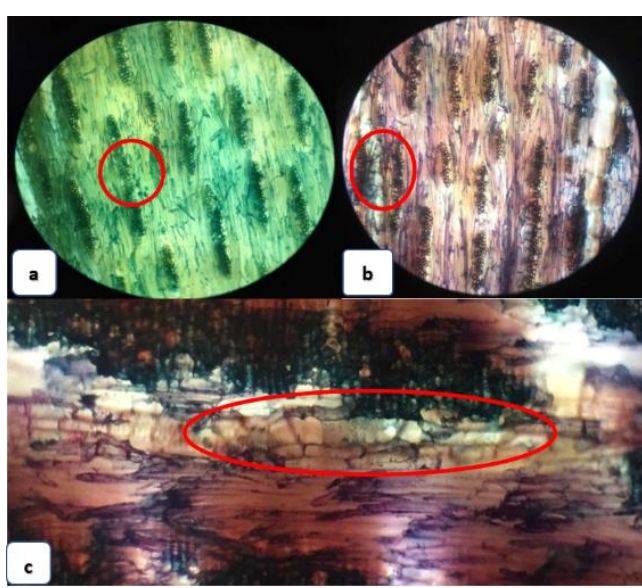

Figura 6. Vista anatómica en corte tangencial de la madera de Cordia alliodora. a. radios parenquimáticos. b. radios multiseriados $\mathbf{c}$. células septadas con radios multiseriados.

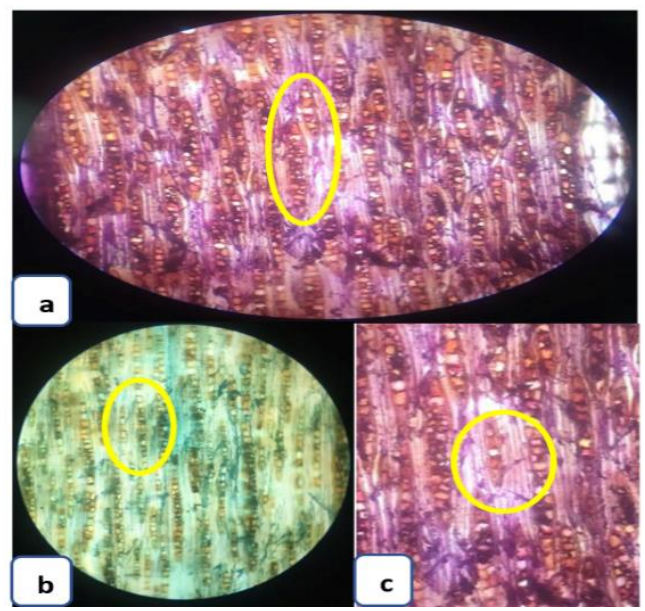

Figura 8. Vista en corte tangencial de la madera de Virola glycycarpa. a. radios uniseriados heterogéneos, $\mathbf{b}$. células procumbentes cuadráticas, $\mathbf{c}$. gomas
Vol. 4, N¹.2, p. 131-146, marzo, 2021

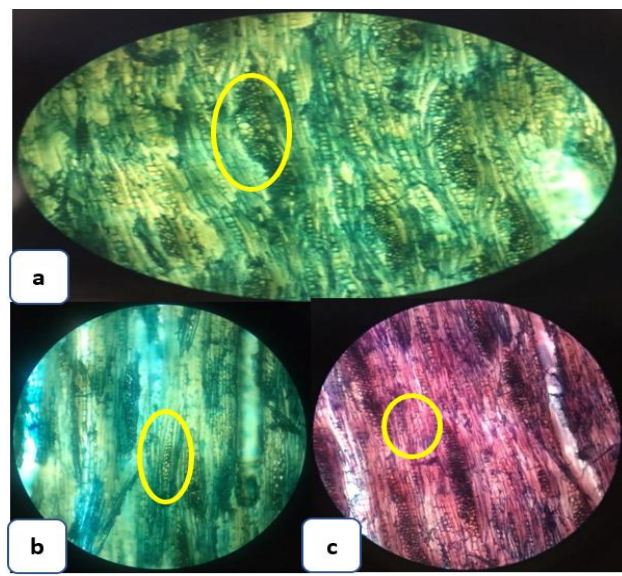

Figura 7. Vista en corte tangencial de la madera Apeiba membranacea. a. radios parenquimáticos multiseriados de células procumbentes. b. radios multiseriados con estructura estratificada. c. células parenquimáticas.

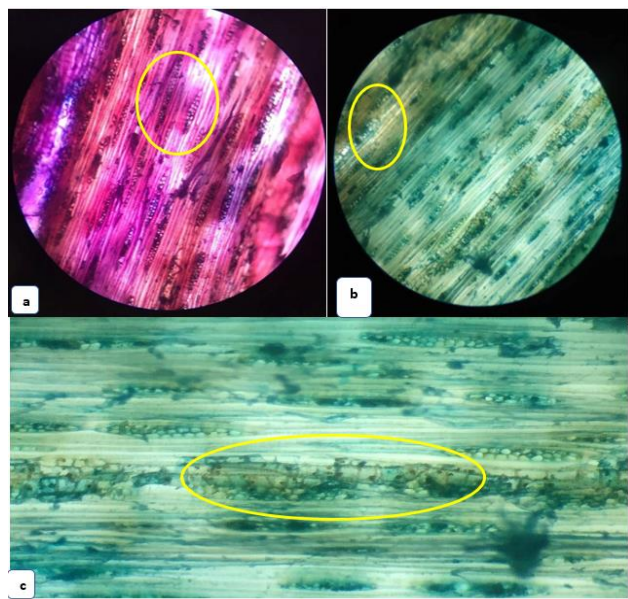

Figura 9. Vista en corte tangencial de la madera de Jacaranda copaia. a. radios parenquimáticos multiseriados, $\mathbf{b}$. células verticalmente $u$ horizontalmente alargadas, cuadradas, no estratificadas, c. presencia de punteaduras.

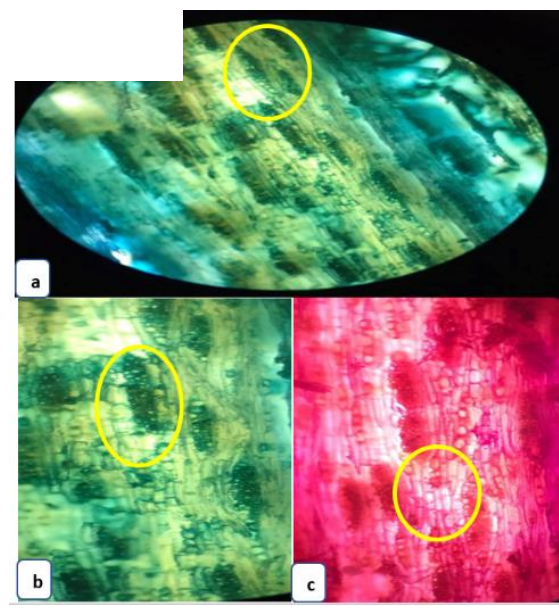


Figura 10. Vista en corte tangencial de la madera de Erisma uncinatum. a. radios biseriados y multiseriados de células procumbentes, b. células procumbentes, cuadradas, c. vaso entrecruzado con células septadas.

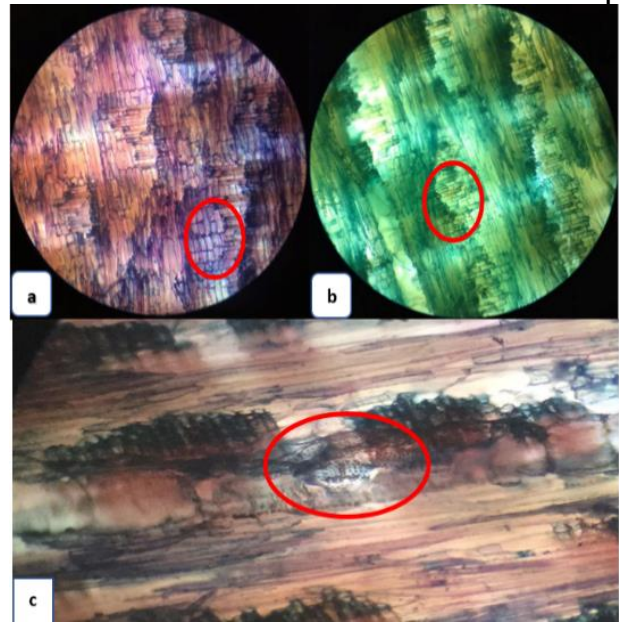

Figura 11. Vista en corte radial de la madera de Cordia alliodora. a. campo de intersección entre fibras y células parenquimáticas.

b. células parenquimáticas. c. parénquima anexo al miembro vascular.

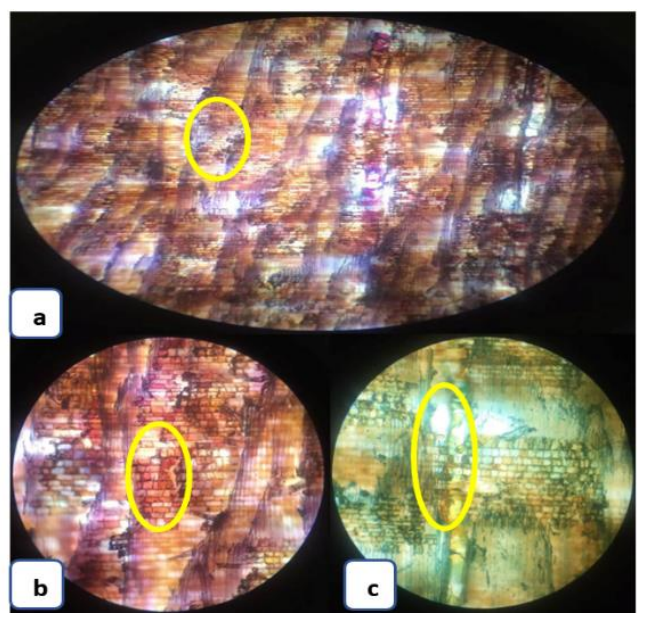

Figura 13. Vista en corte radial de la madera de Virola glycycarpa. a. radios de células erectas o cuadradas, uniseriados y biseriados. b. fibras con punteaduras alargadas, c. sílice

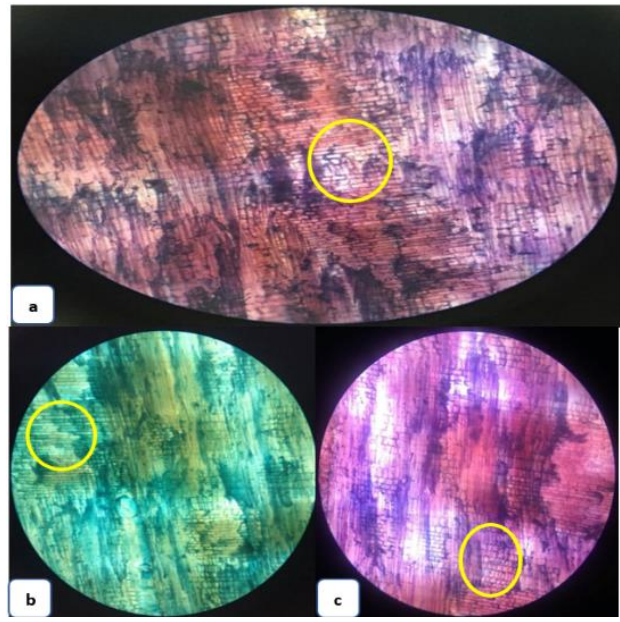

Figura 12. Vista en corte radial de la madera de Apeiba membranacea. a. campo de intersección entre fibras y células parenquimáticas. b. células parenquimaticas. c. radios heterogeneos y multiseriados.

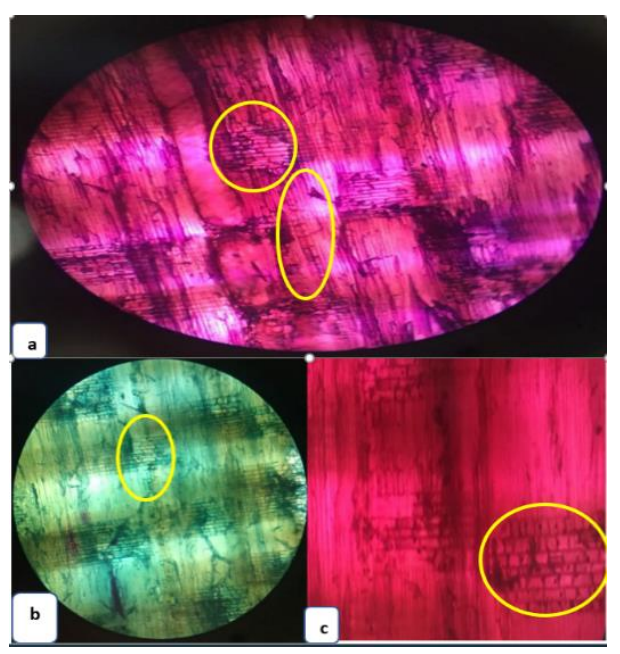

Figura 14. Vista en corte radial de la madera de Jacaranda copaia. a. radios uniseriados y multiseriados, b. células procumbentes, cuadradas y verticales, c. presencia de punteaduras simples ornadas en el vaso. 


\section{Determinación de la densidad}

Tabla 4. Masa, volumen y densidad de las cinco especies estudiadas

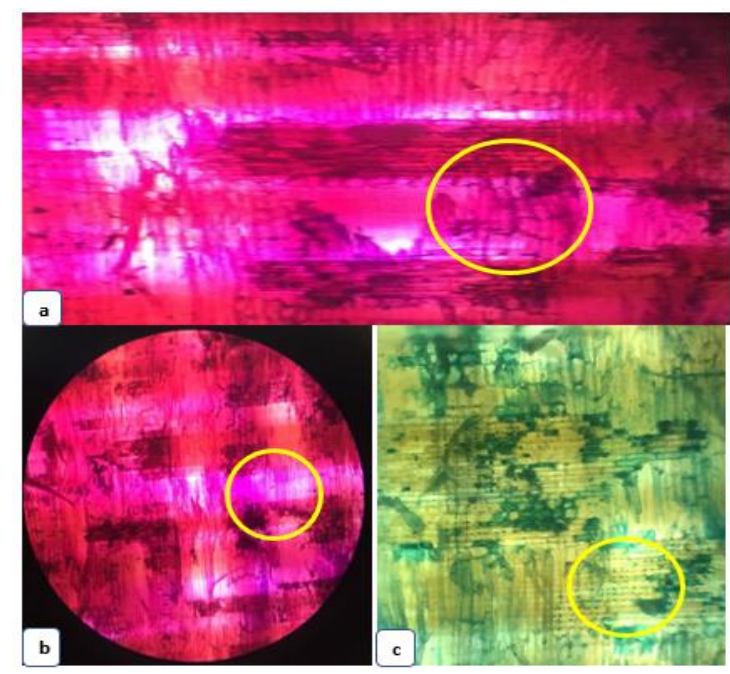

Figura 15. Vista en corte radial de la madera de Erisma uncinatum. a. radios homocelulares de células procumbentes, b. fibras no septadas, c. presencia de punteaduras simples ornadas en el vaso.

\begin{tabular}{cccc}
\hline Especie & Masa $(\mathbf{g})$ & Volumen $\left.\mathbf{( c m}^{\mathbf{3}}\right)$ & Densidad $\mathbf{\rho = g} / \mathbf{c m}^{\mathbf{3}}$ \\
\hline Cordia alliodora & 3,26 & 5,22 & 0,62 \\
\hline Apeiba membranácea & 2,64 & 4,15 & 0,63 \\
\hline Virola glycycarpa & 2,75 & 4,91 & 0,56 \\
\hline Jacaranda copaia & 2,73 & 4,80 & 0,56 \\
\hline Erisma uncinatum & 2,75 & & 0,58
\end{tabular}

Fuente: Equipo técnico (2020)

5. Análisis estadístico de la cantidad de los poros 
Con respecto al número de poros solitarios se observaron diferencias estadísticas entre las especies. Luego de llevar a cabo la prueba de Tukey se observó que las especies se agrupan en cuatro rangos que de menor a mayor van: 1) Virola glycycarpa Ducke con 173 poros y Cordia alliodora con 171 poros solitarios, 2) Apeiba membranácea con 229 poros solitarios, 3) Erisma uncinatum Warm. con 562 poros solitarios, 1) Erisma uncinatum Warm. con 632 poros solitarios (Tabla 5).

Tabla 5. Promedio del número de poros solitarios encontrados en las 5 especies estudiadas (Tukey; $p<0,05$ )

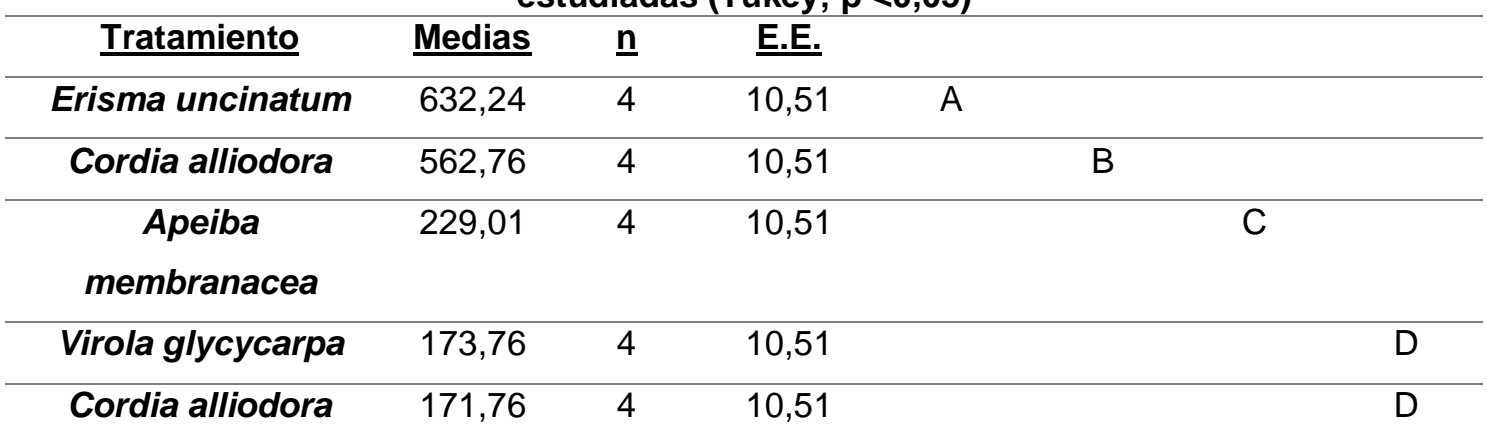

Medias con letras iguales son estadísticamente similares

Respecto al número de los poros múltiples se encontraron diferencias estadísticas entre las especies estudiadas. Luego de aplicar la prueba de comparaciones múltiples de Tukey sobre el número de poros múltiples se pudo observar que las especies se agrupan en cuatro rangos que de menor a mayor número de poros múltiples se ordenan de la siguiente manera: 1) Jacaranda copia con 9 poros y Erisma uncinatum Warm con 4,74 poros, 2) Cordia alliodora con 38 poros, 3) Apeiba membranácea con 61 poros y 4) Virola glycycarpa Ducke con 176 poros. Las especies del rango 4 no presentan diferencias entre ellas (Tabla 6).

Tabla 6. Promedio del número de poros múltiples encontrados en las 5 especies estudiadas (Tukey; $p<0.05$ )

\begin{tabular}{lcccccc}
\hline Tratamiento & Medias & $\underline{\mathbf{n}}$ & $\underline{\text { E.E. }}$ & & & \\
\hline Virola glycycarpa & 176,74 & 4 & 3,30 & A & & \\
\hline Apeiba membranacea & 61,24 & 4 & 3,30 & & B & \\
\hline Cordia alliodora & 38,26 & 4 & 3,30 & & & \\
\hline Jacaranda copaia & 9,01 & 4 & 3,30 & & & D \\
\hline Erisma uncinatum & 4,74 & 4 & 3,30 & & D
\end{tabular}

Medias con letras iguales son estadísticamente similares.

Fernández F. (2019), que estudio Cordia alliodora coincide con los resultados referentes al color de albura y duramen, así como también con el grano recto de la especie. En relación con el sabor Córdoba Foglia, R. (1997) registra que no presenta un sabor definido. Barrance, et al (2003) determinó que la densidad básica varia de 0,34 a 0,64 $\mathrm{g} / \mathrm{cm}^{3}$, además dice que estas variaciones en los valores registrados podrían deberse a 
ciertos eventos como la precipitación, en zonas secas la madera es más pesada en relación con otras que vienen de áreas húmedas los datos de densidad obtenidos en este estudio $\left(0,62 \mathrm{~g} / \mathrm{cm}^{3}\right)$ están dentro de los rangos mencionados.

Arévalo y Londoño. (2005) describen en Colombia las características macroscópicas y organolépticas de Apeiba membranaceae y coinciden con este trabajo en que el olor y el sabor no son distintivos, su grano es recto y la textura gruesa, la transición entre albura y duramen no es marcada, datos que se observan también en el estudio de Rojas, L., León W., (2019).

En relación con los estudios sobre Jacaranda copaia que fueron realizados por Aisalla (2019), las características macroscópicas presentan similitud en base al color que es amarillento pálido, lustre medio con veteado de tipo jaspeado satinado, el grano es recto y textura media a gruesa, existiendo diferencias con el olor que es fragante. Adicionalmente otro punto de coincidencia está en que la mayoría de poros solitarios presenta un parénquima aliforme.

Las fotografías del corte transversal registradas en este trabajo para Erisma uncinatum coinciden con las reportadas por Barrios et al. (2016) en un estudio realizado en Colombia. En relación con las características generales al comparar con el estudio llevado a cabo por Roque et al. (2007) encontramos que hay discrepancias con respecto al color (albura de color blanco amarillento y duramen marrón claro) y textura (gruesa); así como también semejanzas en cuanto a olor, sabor, brillo y grano. En referencia a la descripción de los poros, al revisar el estudio de Pérez (2018) realizado en Perú, se observa que en cuanto a la descripción de los poros, parénquima y radios llegamos a resultados similares.

Con respecto a la densidad de las cinco especies estudiadas, las especies que presentaron mayor densidad fueron Virola glycycarpa Ducke (Sangre de gallina), Jacaranda copaia (Jacaranda), Erisma uncinatum, dureza media Cordia alliodora, Apeiba membranacea. Las especies que presentaron mayor densidad fueron Apeiba membranacea con 0,63 g / $\mathrm{cm}^{3}$, Cordia alliodora (Laurel) con $0,62 \mathrm{~g} / \mathrm{cm}^{3}$. Registran densidades menores Virola glycycarpa Ducke con 0,56 $\mathrm{g} / \mathrm{cm}^{3}$, Jacaranda copaia con $0,56 \mathrm{~g} / \mathrm{cm}^{3}$, Erisma uncinatum con $0,58 \mathrm{~g} / \mathrm{cm}^{3}$

\section{Referencias bibliográficas.}

Aisalla, E (2019). Caracterización anatómica de la madera de cinco especies comerciales mediante tinciones procedentes del Cantón Tena, Provincia de Napo. Tesis para obtener el título de Ingeniero Forestal, Escuela Superior Politécnica de Chimborazo, Riobamba Ecuador. 
Agila, S., Burneo, A., Narváez, L., \& Pucha-Cofrep, D. (2018). Propiedades físicas y características anatómicas de la madera de tres especies de Guayacán al sur del Ecuador. Bosques Latitud Cero, 8(2): 1-15.

Arévalo, R. L. \& Londoño, A. O. (2005). Manual para la identificación de maderas que se comercializan en el departamento del Tolima. Ibagué: Corporación Autónoma Regional del Tolima e Impresiones Conde.

Barrance, A., Beer, J., Boshier, D., Chamberlain, J., Cordero, J., Detlefsen, G., . . . Pennington, T. (2003). Arboles de Centro America. Costa Rica: Turrialba.

Barrios P, N. M., León Hernández, W. J., \& Pacheco A, C. E. (2016). Determination of wood anatomical quantitative features by means of geographic information technology. Pittieria, (40), 54-93.

Comisión Panamericana De Normas Técnicas. (1972). Maderas método de determinación del peso específico aparente [en línea]. [Consulta: 10 diciembre 2020]. Disponible en: http: www.copant.org/index.php/es/.

Córdoba Foglia, R. (1997). Características, propiedades y usos de la madera del laurel (Cordia alliodora). Revista Forestal Centroamericana Volumen 6, número 20 (julioseptiembre 1997), páginas 18-23

Crespo, J. (2011). Notas sobre anatomía de maderas en relación al secado. Revista Mexicana de Ciencias Forestales, 1: 42-53.

Di Rienzo J. A., F. Casanoves, M. G. Balzarini, L. González, M. Cuadroda, C. W. Robledo. InfoStat versión (2012). Grupo InfoStat, FCA, Universidad Nacional de Córdoba, Argentina. URL http://www.infostat.com.ar.

Feijoo, C. E., Pucha, A. D., \& Ramon, D. D. (2019). Guía para cortes anatómicos de la madera. Loja-Ecuador. 9-45.

Fernández, F. (2019). Propiedades organolépticas, físicas y mecánicas de la madera de Cordia alliodora (R. y P.) Oken de parcelas agroforestales en Jaén, Cajamarca. Revista Científica Pakamuros, 7(1), 80-91. https://doi.org/10.37787/pakamuros-unj.v7i1.78

Rojas, L., León W., (2019). Anatomía de la madera de 25 especies de malvaceae en Venezuela. Revista científica Pittieria, 8-33. http://erevistas.saber.ula.ve/index.php/pittieria/article/viewFile/15780/21921926897 \#page $=8$

Martínez. J., Borja de la Rosa, A. \& Velasco, M. (2005). Características tecnológicas de la madera de palo morado (Peltogyne mexicana Martínez) de Tierra Colorada, Guerrero, México. Revista Chapingo Serie Ciencias Forestales y del Ambiente, 11(1): 78-82. 
Pérez, F. (2018). Estructura anatómica y claves de identificación de cinco especies maderables del Perú. Tesis para obtener el título de Ingeniero Forestal, Universidad Nacional Agraria, Lima Perú.

Roque, R., Gómez, M., \& Rivero, J. (2007). Clave de identificación macroscópica para 22 especies maderables de Bolivia. Revista Forestal Venezolana, 51(2), 179-193.

Rowe, N., \& Speck, T. (2005). Plant growth forms: an ecological and evolutionary perspective. New phytologist, 166(1), 61-72.

Sauter, J. J., \& van Cleve, B. (1994). Storage, mobilization and interrelations of starch, sugars, protein and fat in the ray storage tissue of poplar trees. Trees, 8(6), 297-304.

Sperry, J. S. (2003). Evolution of water transport and xylem structure. International Journal of Plant Sciences, 164(S3), 115-127.

Wiedenhoeft, A. C., \& Miller, R. B. (2005). Structure and function of wood. Handbook of wood chemistry and wood composites, 9-33. 


\section{PARA CITAR EL ARTÍCULO INDEXADO.}

Salazar Castañeda, E. P., Carpio Coba, C. F., Noboa Silva, V. F., \& Verduga Coyago, I. V. (2021). Caracterización botánica y anatómica de 5 especies maderables de bosque secundario en el sector el Rocío, del Cantón Loreto, provincia de Orellana . ConcienciaDigital, 4(1.2),

https://doi.org/10.33262/concienciadigital.v4i1.2.1584

\section{Ciencia}

El artículo que se publica es de exclusiva responsabilidad de los autores y no necesariamente reflejan el pensamiento de la Revista Ciencia Digital.

El artículo queda en propiedad de la revista y, por tanto, su publicación parcial y/o total en otro medio tiene que ser autorizado por el director de la Revista Ciencia Digital.
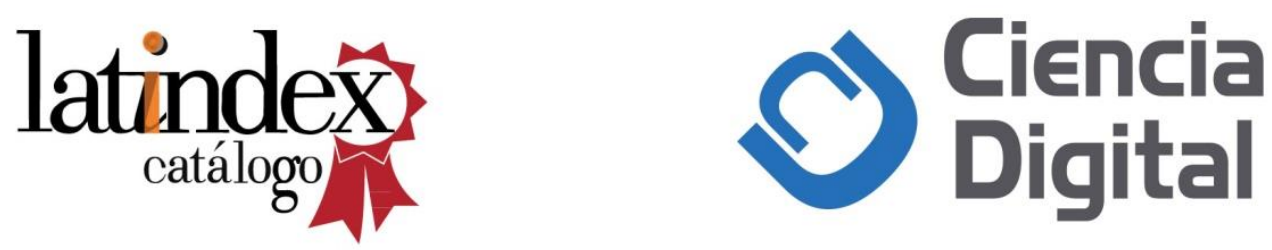